\title{
Collaborative Writing Across Multiple Artifact Ecologies
}

\author{
Ida Larsen-Ledet \\ Dept. of Computer Science, \\ Aarhus University, Denmark \\ ida.11@cs.au.dk
}

\author{
Henrik Korsgaard \\ Dept. of Computer Science, \\ Aarhus University, Denmark \\ korsgaard@cs.au.dk
}

\author{
Susanne Bødker \\ Dept. of Computer Science, \\ Aarhus University, Denmark \\ bodker@cs.au.dk
}

\begin{abstract}
Research focusing on how collaborative writing takes place across multiple applications and devices and over longer projects is sparse. We respond to this gap by presenting the results of a qualitative study of longer-term academic writing projects, showing how co-writers employ multiple tools when working on a common text. We identify three patterns of multi-application collaboration as well as four common types of motivations for transitions between applications. We also extend existing taxonomies of collaborative writing by proposing a categorization of the functions served by the text as object and backbone of the collaboration. Together, these contributions offer a framing for understanding transitions within and across artifact ecologies in work around a common object. Our findings highlight ways in which features like concurrent editing may in fact challenge the collaborative writing process, and we point to opportunities for alternative application models.
\end{abstract}

\section{Author Keywords}

Collaborative writing; artifact ecologies; text function

\section{CCS Concepts}

-Human-centered computing $\rightarrow$ Empirical studies in collaborative and social computing; User studies;

\section{INTRODUCTION}

Collaborative writing has been an important research topic in Human-Computer Interaction and Computer-Supported Collaborative Work for decades. There have been vast technological developments, from the early text processing tools in the 1980's providing basic means for communication and shared viewing [20,24], to current text editors with capabilities for crowd sourcing [3] or automatic prediction of needs for changes [26], and well-known collaborative editing platforms like Google Docs [28], Overleaf [54], and GitHub [27]. The crucial work by Gibbs and Ellis [19] on operational transformation (OT) has made real-time collaboration possible and key work on communication support and awareness have influenced the design of today's platforms.

Permission to make digital or hard copies of all or part of this work for personal or classroom use is granted without fee provided that copies are not made or distributed for profit or commercial advantage and that copies bear this notice and the full citation on the first page. Copyrights for components of this work owned by others than ACM must be honored. Abstracting with credit is permitted. To copy otherwise, or republish, to post on servers or to redistribute to lists, requires prior specific permission and/or a fee. Request permissions from permissions@acm.org.

CHI '20, April 25-30, 2020, Honolulu, HI, USA.

Copyright is held by the owner/author(s). Publication rights licensed to ACM. ACM ISBN 978-1-4503-6708-0/20/04 ...\$15.00.

http://dx.doi.org/10.1145/3313831.3376422
Despite this development and how commonplace collaborative writing is, some areas warrant further study. Few have looked into how collaborative writing happens across multiple applications and devices: How co-writers negotiate, deal with, and use multiple applications in the writing process; and why and how this influences the process and outcome. Collaborative writing is often studied from the angle of a single tool and/or document in projects lasting only hours, days, or weeks at most, which does not reflect how collaborative writing is currently practiced. We present a qualitative study of co-located and distributed academic writing projects lasting for extended periods of weeks, months, and years, involving co-production of a joint report, research paper, or book.

First, we examine the collection of applications mediating the writing process. Collaborators use and depend on a number of different applications, e.g., for sharing documents and text, and for coordinating and communicating throughout the process. They use particular applications for doing layout or producing figures, etc. These applications are partly chosen with a basis in past experiences, partly due to functional needs, and partly based on co-writers' personal preferences [69]. We use the concept of artifact ecology [35, 7, 60] to map the collection of collaborative writing tools and analyze how coauthors use and appropriate these. Based on this analysis, we identify three patterns to describe strategies for collaboration with and across multiple applications: collaborative home, repository, and hand-over. Second, we identify and discuss four common types of motivations for application transitions occurring throughout the writing project: functional, personal space, aesthetic and user experience, and communication. Application transitions refer to collaborators switching between multiple applications when writing, e.g. moving text from a shared document into a personal text editor, transitioning from writing to layout applications, or tracking down co-authors through in-editor comment features and communication applications. These transitions are rarely motivated by utility and availability alone. Need for personal space and collaborative "noise", such as jumping text, are additional examples of reasons for transitions between applications in collaborative writing. Third, in the kind of collaborative writing projects examined here, the text itself serves multiple roles in the writing process, something not addressed in existing taxonomies of collaborative writing. As pointed out by Neuwirth et al., "[...] experienced writers typically engage in many acts of writing (e.g., jotting down ideas, drawing) that bear no direct relation to the text product, but serve as inexpensive, intermediate external representations to remind writers of their plans for audience, purpose, and procedure as well as content." [48, 
p. 540]. Building on the interviews, analysis, and related work $[56,33]$, we propose a categorization of the roles played by the text as common object of work into seven functions that may overall be divided into interim material outcome, locus of coordination, and epistemic object.

The three focus areas above contribute to our understanding of collaborative writing as a practice that is dynamic and complex in the applications it involves and the roles applications and text play in the process. We see application transitions as a useful addition to existing work on artifact ecologies, as well as consolidating prior work into a coherent model depicting personal artifact ecologies $[35,7]$ in relation to potential and aligned artifact ecologies $[60,53]$ (see figure 1). The identified text functions extend existing taxonomies on collaborative writing, in particular Posner and Baecker's [57] work on document control styles and writing strategies, as well as others' contributions to the latter [43, 70], and Wang et al.'s [66] identification of text production patterns.

Several of the application transitions we have observed move away from the primary collaborative platform. Our findings suggest that application symmetry (everyone having the same level of access, features, notifications) and real-time editing challenge aspects of (collaborative) writing; for example causing disruptions that compel co-writers to periodically abandon the common object of work. We see significant challenges in supporting collaborative writing across artifact ecologies rather than contained within individual tools, with transitions largely happening through copy-pasting. This points to the exploration of document- and activity-centric [37, 34] alternatives for application design as an avenue for future work.

\section{ARTIFACT ECOLOGIES AND COLLABORATIVE WRITING}

The focus on artifact ecologies in collaborative writing necessitates a brief introduction to the concept of and key perspectives on artifact ecologies and common objects. This provides a foundation for presenting the findings from the study.

\section{Conceptualizing Artifact Ecologies}

The concept of artifact ecologies is drawn from works discussing and conceptualizing multiple artifacts and their relation to concrete practices. The term is one of several examining how (interactive) artifacts, e.g. devices and software applications, are part of a specific ecology that influences how people make sense of, combine, substitute, and appropriate technology (e.g. information ecologies [46], personal artifact ecologies [35, 7], community artifact ecology [9], digital assemblies [61], constellations of technologies [60]). While the background and terminology vary, we see the concepts as closely related and complementary. As a unified concept, the notion of artifact ecologies allows for examining the multiple interactive artifacts in use throughout, e.g., a collaborative writing process. In particular, it highlights the multiplicity and dynamics of computer-mediated collaborative writing, including the interplay between the personal and the shared, and how they shape the ecology and its use.

Artifacts typically originate within a personal artifact ecology defined as the collection of artifacts a person owns, has access to, and uses [35, p. 201]. Familiarity is key, and artifacts are understood in relation to common uses and complement other artifacts within the ecology. A personal artifact ecology is dynamic and changes as new needs and desires arise and/or when people learn from - or collaborate with — others [8]. In collaborative writing, the potential artifact ecology is the sum of co-writers' personal artifact ecologies, from which they negotiate an aligned artifact ecology consisting of the tools and applications to be used in the work [60]. Rossitto et al. [60] use the term orchestration to describe the (meta-) activity of aligning the group's work ecology and describe how alignment of the ecology happens both at the onset of the group's formation and in relation to different contextual, temporal, and task-specific constraints throughout the work. Oulasvirta and Sumari [53] discuss similar activities in multi-device management and configuration, however, focusing on the pragmatics of setting up in a physical setting, rather than negotiating.

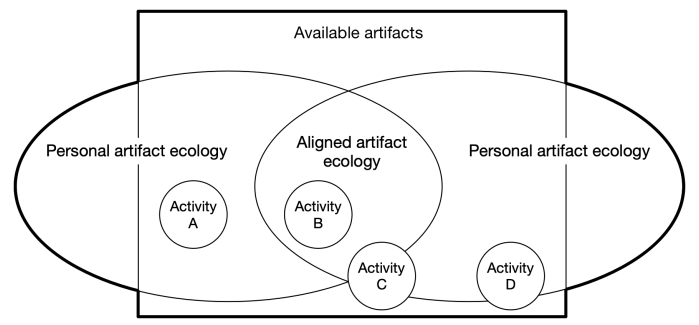

Figure 1: Overview of artifact ecologies in collaborative writing.

In figure 1, we integrate work on artifact ecologies into a model that shows the overlaps between personal artifact ecologies, the potential artifacts, and the aligned parts of the ecologies, as well as activities that are carried out using these artifacts. Figure 1 depicts the simplest possible collaboration structure but generalizes to the more complex patterns common in collaborative writing. In figure 1, the potential ecology (bold) is a combination of co-writers' personal artifact ecologies and any additional tools available at the time and place of the activities (see also $[53,18]$ ). The active parts are the particular artifacts in use in a given activity. Although Rossitto et al. [60] focus on how the aligned ecology is a result of the potential ecology - that is, the available and familiar artifacts to the users we find that activities and tasks do not always happen within the aligned ecology alone (elaborated in the findings section). Work towards the shared goal can happen using artifacts from one participant's ecology alone, e.g. when this co-writer is the only one with access or the necessary expertise to use an artifact (figure 1, activity A). Work that requires that all the participants use an instance of the same tool happens within the aligned ecology, e.g. writing in a shared document or communicating (figure 1, activity B). Activities can also happen at the intersection of ecologies (figure 1, activities $\mathrm{C}$ and D), e.g. reviewing and editing a shared document displayed on a projector in a meeting space. While the model captures trivial observations (e.g. Raptis et al. [58] propose a similar model from a single-user perspective), the present work is particularly concerned with how different artifacts support and introduce transitions between technologies spanning multiple ecologies. This is part of the analysis and findings we present. 


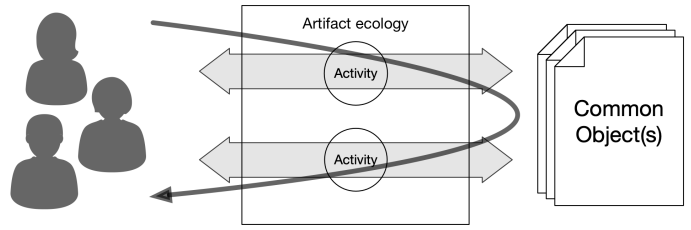

Figure 2: The collaborative activity is mediated by the artifact ecology and focuses on the common objects at play in the creation of the text.

The combined model does not capture how the ecologies change as people become familiar with other tools through collaborating over time. Although development over time is an important aspect of artifact ecologies (see [46, 7, 9]), integrating this is outside the scope of the present work.

\section{Common Objects}

With inspiration from activity theoretical HCI [6] and instrumental interaction [2] we see human activity as mediated, by artifacts and artifact ecologies. Writing is, as we analyze and discuss it in detail later, mediated by a variety of artifacts from pens to word processors, and even spellcheckers and formatting tools. Users are simultaneously working, collaborating, and focusing on the objects of their activity [6] (see figure 2). Borrowing terminology from Robinson [59] or Heath and Luff [30] these objects are held in common. Heath and Luff address ways of collaboratively accessing objects or "[...] a range of capabilities from the simple exchange of objects, through the sharing of objects and to common views of the same object." [30, p. 361]. Such objects are always intermediary in several meanings of the word:

First, they are a result of the current state of the activity as the object moves from (shared) materials to product, as discussed by Engeström [21] and by Sørgaard [62]. This is the case, e.g., when bits of text are edited together into a coherent, shared document, or when data is turned into a table. Second, the common objects are epistemic in the sense that they contain and represent for the user an idea of the end product (or intermediate products along the way). This can be an idea of the contents, of the message, etc., or it can be the mere fact that the paper needs to be, e.g., six pages long. Co-writers are mutually responsive to one another and have a more or less shared goal that they all identify (with), while they coordinate their plans of action and intentions. Tenenberg et al. [64] discuss how the goals and intentions of each participant partly incorporate the goals and intentions of the other collaborators. Finally, since such activity is complex and collaborative, every once in a while the co-writers, alone or together, have their activity focused on objects that are parts of the whole, or interim steps towards the whole, such as when drafting an outline without worrying about the text or the format. In this manner the common objects form the backbone of the communication and coordination happening in the co-writing process, or in the terms of Nicolini et al. [49], they are the fundamental infrastructure of the activity.

A large body of work has addressed the roles of documents and other artifacts in collaborative activities [33, 56, 23, 55]. These roles include communication $[56,51,55]$, coordination
$[56,33,55]$, and consolidating the work [56, 23]. Other work has studied how documents and other artifacts are used across devices [51,60]. While informative, these works do not address or capture the nuances of (collaborative) writing.

\section{Collaborative Writing}

Research from the early 2000s indicates that academic writers, at the time, did not use writing tools designed for collaboration, even when available [36, 50]. Based on an interview study and an online survey, Kim and Severinson Eklundh [36] and Noel and Robert [50] found that co-writers stayed with their preferred word processors, even though these were designed mainly for solitary use. Coordination by these writers happened mainly through e-mail and face-to-face meetings. Kim and Severinson Eklundh [36] note that even though the tools used by co-writers had features for collaboration, such as comments, these were not used. In recent work, Birnholtz and Ibara [4] and Wang [65] provide an updated view of co-writers' writing practices. Wang's findings show a more diverse use of tools including designated collaboration tools and features. Both also discuss synchronous writing as more common than what has previously been described $[16,36,15,50]$.

We have previously discussed (text) state as a dimension of collaborative writing [42], implying a coordinating role of the text which has inspired the notion of text function presented in this paper. That work additionally showcased socially sustaining behaviors in the writing process, in parallel with Birnholtz and Ibara's [4] description of how features for change awareness can impact relationships between co-writers. The relational side of collaborative writing is further explored by Wang et al. [67] and Allen et al. [1], both emphasizing technology's effect on the production of shared writing. Mitchell et al. [45] present a study of collaborative writing by school children, focusing on ownership and control.

Neuwirth et al. [48] note that collaborative writing involves many activities other than synchronous writing and that these are not always supported by the writing tool. Kim and Severinson Eklundh [36] report how co-writers would typically agree on a writing tool at the start of the process but may individually use additional tools. Boellstorff et al. [10] recount the emergence of a toolkit of multiple applications to support different needs during the collaborative writing process. These observations are closely related to those discussed by Rossitto et al. [60], and stress a need to focus on the artifact ecologies of collaborative writing and how these influence the writing.

\section{Taxonomies of Collaborative Writing}

Several works present taxonomies of collaborative writing, parts of which are summarized in table 1. Allen et al. [1] present a typology of shared-document collaboration. Posner and Baecker [57] outline a taxonomy that includes four categories: Roles refer to the kind of work each individual is doing, such as writing or editing, and activities are a set of sub-activities such as planning or writing. Writing strategies and document control methods describe how the work and the control is distributed throughout the document and the writing process, such as through joint writing and shared control. Lowry et al. [43] include additional writing strategies, such as the reactive writing strategy. While Posner and Baecker 
Table 1: Writing Strategies, Document Control Styles, and Text Production Patterns from related work, with the addition of Text Functions as developed within this paper. Activities, roles, and work modes from related work have been omitted as they are not used directly in the analysis.

\begin{tabular}{|c|c|}
\hline Category and Type & Description \\
\hline $\begin{array}{l}\text { WRITING STRATEGIES } \\
\text { Single writer [57] } \\
\text { Scribe [57] } \\
\text { Sequential writing [43] } \\
\text { Parallel writing [43] } \\
\text { - Horizontal [43] divide and conquer [70] } \\
\text { - Horizontal [43] cooperative revision [70] } \\
\text { - Stratified division [43] } \\
\text { Joint writing [57] } \\
\text { Reactive writing [43] } \\
\text { Mixed mode [43] } \\
\text { Consulted [57] }\end{array}$ & $\begin{array}{l}\text { One person writes the document based on group discussions. } \\
\text { One person writes down the group's thoughts during a meeting. } \\
\text { The writer role is passed from person to person, one at a time. } \\
\text { Each person is responsible for a different part of the writing. } \\
\text { Writers write their own parts and rarely edit each other's text. } \\
\text { Writers write their own parts, but freely edit each other's text. } \\
\text { Each participant plays a particular role based on their expertise. } \\
\text { The group jointly decides on the wording and sentence structure. } \\
\text { Writers react and adjust to each other's work in real time. } \\
\text { A mix of multiple strategies. } \\
\text { The writing is guided by a person who does not take part in the writing. }\end{array}$ \\
\hline $\begin{array}{l}\text { DOCUMENT CONTROL } \\
\text { Centralized [57] } \\
\text { Relay [57] } \\
\text { Independent [57] } \\
\text { Shared [57] }\end{array}$ & $\begin{array}{l}\text { One person is in charge of the document throughout the project. } \\
\text { Control passes between one person at a time. } \\
\text { Individuals maintain control of separate parts of the document. } \\
\text { Multiple people are in charge of the document with equal privileges. }\end{array}$ \\
\hline $\begin{array}{l}\text { TEXT PRODUCTION } \\
\text { The outline pattern [66] } \\
\text { The example pattern [66] } \\
\text { The best-of-each pattern [66] }\end{array}$ & $\begin{array}{l}\text { Work is distributed through the creation of an outline in the document. } \\
\text { The content of a similar type of text is replaced with new content. } \\
\text { Each person writes the full text, and the group selects the best of each. }\end{array}$ \\
\hline $\begin{array}{l}\text { TEXT FUNCTIONS [PRESENT CONTRIBUTION] } \\
\text { Material } \\
\text { Interim outcome } \\
\text { Outline } \\
\text { Plan } \\
\text { Division of work } \\
\text { Pointing ahead } \\
\text { Reflexivity } \\
\text { Reactivity }\end{array}$ & $\begin{array}{l}\text { The text is the material being worked on. } \\
\text { The text allows interpretation about its current state. } \\
\text { The text functions as an outline of the text-to-be. } \\
\text {.. an overview of work to be done. } \\
\text {.. a reminder of how work is divided among co-writers. } \\
\text { The text is an embodiment of the common vision. } \\
\text { The text allows reflection on and alignment of the text-to-be. } \\
\text { The text enables co-writers to become involved in each other's work. }\end{array}$ \\
\hline
\end{tabular}

describe joint writing as involving group decisions even on "minute components of the text" [57, p. 134], Lowry et al.'s reactive writing is presented as co-writers "reacting to and adjusting to each other's changes and additions" [43, p. 78], such as when co-writers review a section as it is being written and respond by writing new sections of their own. Both strategies are hence considered here. In more recent work, Yim et al. [70] identify a number of writing styles, including two versions of Lowry et al.'s horizontal parallel writing (table 1): divide and conquer and cooperative revision. We use the latter to refer to asynchronous writing, and reserve reactive writing for cases with simultaneous writing. Wang et al.'s recent work [66] identifies strategies for text production, also included in table 1. Finally, ways to implement these concepts have been explored [24, 29, 47, 63], such as by allowing activities and roles to be specified and managed alongside the writing [68].

We find it surprising that none of this prior work addresses the text itself. Through our current study we have become aware of the multiple levels of meaning that the text holds for cowriters during their work. At any given moment, the function served by the text is highly significant to how work on and around the text is carried out. For example, the text may serve as a coordination tool for dividing up the work or achieving an awareness of who is currently working and on what. Hence we introduce the notion of text functions, summarized along with related work on writing taxonomies in table 1 , which we elaborate further in the findings.

\section{STUDY AND METHODOLOGY}

Our findings come from interviews with researchers and Master's degree students involved in collaborative writing projects. The projects around which the interviews centered comprised joint master's theses, a student project, scientific papers, books, and a magazine article. The interviews covered a total of 18 different projects, with five participants being involved in more than one of these. The writing part of these projects lasted between 1 week and 2.5 years (see table 2 for an overview). The study was approved by Aarhus University's research ethics committee and participants have been informed and have given full consent per European Research Council ethical guidelines.

The questions posed to participants focused on both practical and social aspects of the collaborations, such as the applications used, strategies for text production and division of work, ways of editing text written by co-writers, draft writing, and decision-making. Each interview centered around a particular recent writing project undertaken by the participant(s) but we did not enforce a strict focus on only those cases when participants included other examples. The conversation was allowed to flow naturally, but at opportune moments participants were steered towards topics of interest. Each interview lasted between 50 and 86 minutes, for an average of 64 minutes.

The interviews with students' groups were conducted as group interviews with all participating members present, as we valued the potential for participants' collective recollection and 
Table 2: Study participants: Collaboration history and duration. St = students, $\mathrm{Re}=$ researchers. \# means that the group worked solely distributed.

\begin{tabular}{l|l|l|l|l|l|l|l|l|l|l|l|l|l|l|l|l|l|l}
\hline ID & G01* & G02* & G03* & G04* & G05* & G06 & G07* & G08\# & G09\# & G10 & G11\# & G12 & G13 & G14 & G15\# & G16* & G17 & G18 \\
Type & St. & St. & St. & St. & St. & Re. & Re. & Re. & Re. & Re. & Re. & Re. & Re. & Re. & Re. & Re. & Re. & Re. \\
\hline Size (\# interviewed) & $4(3)$ & $2(2)$ & $3(3)$ & $3(3)$ & $2(2)$ & $4(2)$ & $4(2)$ & $6(2)$ & $5(2)$ & $2(2)$ & $3(2)$ & $2(2)$ & $4(2)$ & $3(1)$ & $3(1)$ & $5(3)$ & $40(1)$ & $2(2)$ \\
Collab. history (years) & $1-5$ & 1 & $1-5$ & 4 & $1-5$ & $0-5$ & $0-1$ & $0-12$ & $0-6$ & $<1$ & $2-10$ & 2 & $1-6$ & - & $0-3$ & $0-27$ & - & 13 \\
\hline Duration (weeks) & 34 & 17 & 19 & 19 & 24 & 5 & 5 & 13 & 4 & 18 & 19 & 2 & 3 & 1 & 56 & 28 & 32 & 183 \\
\hline
\end{tabular}

for them to spur each other on in the conversation [25, 38]. By contrast, the interviews with groups of researchers were conducted individually. The rationale for this distinction was a concern that the power and seniority imbalance in relationships between researchers working together in a professional environment (e.g. PhD students and supervisors) would inhibit participants from speaking openly [14]. While we acknowledge that Master's degree students have equally complex relationships with collaborators, we weighed collective recollection over this concern because of the more equal nature of the students' relationships. Ways of accounting for potential bias in the student groups included asking about hypothetical scenarios and experiences in other collaborations, giving the participants a way of expressing viewpoints and sharing experiences without necessarily criticizing collaborators.

The follow-up interviews (marked $*$ in table 2) elaborated on topics from the initial round of interviews. They focused on collaboration in Google Docs, due to an overlap with an additional research focus. All follow-ups were conducted as group interviews to support collective recollection [25, 38]. This was further supported by consulting a document co-authored by the group. The average duration of the follow-up interviews was 58 minutes, ranging between 39 and 75 minutes.

\section{Participants}

The 32 participants comprised 13 Master's degree students and 19 researchers. In most cases only some group members were available for interviews, and in the case of G16 one of the group members only participated in the follow-up interview. In table 2 the number of group members participating in the study is indicated in parentheses next to the group size. The researcher participants were of ten different nationalities and were employed at four different universities, while all student participants were Danish and studied at two different universities. 9 of the participants identified as female, the rest as male. A majority of the groups were co-located during most of the writing, with four groups working distributed (marked \# in table 2). Participants were recruited via social media postings, face-to-face contact, emails to various departments at the authors' university, as well as through personal contacts and contacts of participants.

\section{Analysis}

The first round of interviews was transcribed and analyzed through bottom-up thematic coding and meaning condensation [41]. We included both semantic and latent themes [11] in the coding. After coding all transcripts we manually clustered the codes into overarching themes that were prevalent across interviews. The follow-up interviews were not transcribed but codes were noted with time stamps and annotated, since these interviews were not analyzed to provide new codes but to supplement insights from the first round of interviews.

From the above we identified patterns in the ways that cowriters and content move across artifact ecologies. We use pattern in relation to collaborative technologies. As opposed to a complete vocabulary [17], patterns address an intermediatelevel abstraction of a socio-technical situation, capturing possibilities and bottlenecks of that situation. This understanding is based on discussions by, e.g., Hermann et al. [32] according to whom patterns refer to the interplay between a technical system and users as well as users' tasks and organizational constellations. We use the notion to aid the discussion of our findings and to open up for future generative outcomes [17].

\section{FINDINGS}

We start by presenting the artifact ecologies of the interviewed groups, based on figure 1. The types of tools included in such a writing ecology are outlined and we consider participants' motivations for including these tools, in particular tools with overlapping functionalities. This descriptive step is necessary before characterizing patterns of transfer of content between applications and motivations for these. In the subsequent section, we then turn out attention from the tools to the content and to the role of the text in collaborative writing as an intermediate artifact and common object.

\section{Mapping the Ecologies of Collaborative Writing}

The applications and services used by the participants cluster around the primary functions of writing, layout, communication, and file and version management. The tools used can be divided into roughly three categories: a) Asynchronous locally installed word processors and desktop publishing applications, such as Microsoft (MS) Word and Adobe InDesign. When these are used as the main tool, documents are shared through a shared drive and/or via email. b) Asynchronous locally installed LaTeX editors, such as TeXstudio, combined with version control systems, like SVN and Git. The version control systems allow the publishing (pushing) of chunks of changes (commits) to a repository, for instance GitHub or a locally hosted one. The editors have no collaboration capabilities aside from the sharing and tracking of changes (including comments accompanying the commits). c) Real-time web-based text editing applications with built-in support for collaboration. Among our participants these include Google Docs (and Drive), for rich text editing, and Overleaf and ShareLaTeX (now Overleaf) for writing LaTeX documents. The writing projects deployed different approaches to writing and collaboration on a project level (see table 2), with three patterns for supporting collaboration emerging from the interviews: 
Collaborative Home: A majority of the projects $(n=12 / 18)$ employ a collaborative home pattern, where the co-writers write within an online platform that persists and synchronizes the text across multiple clients (e.g., Google Docs or Overleaf). In this pattern there is a high degree of tool and content symmetry, as the collaborators share both the text editor (interface) and how the content is rendered, in real-time. Document control (see table 1) is usually independent, or centralized in the case of a PhD student working with their supervisor and perhaps other collaborators. We encountered only few cases of relay control, and shared control was rare [42].

Repository: A few projects use a repository pattern for collaboration $(n=5 / 18)$. Here the collaborators decide on a common service for persisting and synchronizing files across computers, either automatically or through user-initiated uploads or commits (e.g. using Dropbox or Git). Depending on file formats, this approach offers the possibility to separate the text editor used by each co-writer from the common text (tool asymmetry). Depending on the synchronization model, it is sometimes not possible to work in the same file simultaneously, in which case co-writers often used version control features or negotiated different ways of organising the writing process around merge and versioning issues. Text symmetry (all writers having the same version) is maintained to varying degrees depending on the file sharing platform: When a document is saved directly to a Dropbox, co-writers can maintain a high degree of symmetry, while symmetry is lower, e.g., in the case of a group storing the document in Google Drive and manually downloading and uploading document versions.

Hand-over: A single project used a hand-over pattern with relay control where the co-writers decided on a file format and then exchanged files via email or similar. This pattern shares the potential for tool asymmetry (depending on file format) with the Repository pattern, but may also introduce complete text asymmetry, as there is no centrally available maintained version of the text.

\section{Transitioning Between Multiple Tools}

Participants' aligned ecologies (figure 1) arose from a mix of co-writers' prior experiences and negotiations (see also Rossitto et al. [60]), as well as familiarity and individual preferences for text format (e.g., formatted text or LaTeX) or application features, weighed against advantages and disadvantages. One group started in Google Docs, mainly due to a limit to how many can collaborate in the unpaid version of ShareLaTeX, but moved into ShareLaTeX once they figured out a work-around. Whereas writing tools were subject to early alignment, communication tools were more fluent and subject to ongoing negotiation based on availability, e.g. seeing someone online in a chat application or trying email first and then direct messaging afterwards.

The aligned ecology is rarely the ecology or the only applications used throughout the writing project (see also $[10,36]$ ). Participants for example reported using additional text editing applications briefly throughout the process. One participant's statement highlights this application multiplicity:
1: "I will sit in [MS] Word for a bit and also [Apple] Pages from time to time, just to sitfor myself. Then I will insert [the text] into Google Docs, when I am done with my bit. But I also work directly in [Google Docs] at times." (G02)

Alongside tools used for writing and formatting, all groups reported using multiple tools for coordinating the writing process and collaborating around the document(s) held in common. In addition to their intended uses, applications and features were used in alternative ways to coordinate and communicate. For example, chat applications were used to exchange files and bits of text during parallel writing, or with supervisors during consulted writing. Comments and built-in messaging were used to communicate between collaborators for light banter or for grabbing a co-writer's attention.

Transitions among both writing and communication tools are linked with preference and availability (installed, free, already open), but also associated with differences in the devices and platforms individual co-writers use. Some transitions are substitutions of applications or features available in the aligned ecology, whereas others represent additions brought in to cover specific needs in the process. All of the groups working in Google Docs would, for instance, switch to another tool, such as ShareLaTeX or MS Word, to format the text and add references. Despite the intention of some of these groups to use the new application solely for formatting, rendering, and references, participants described how they would often make changes to the text after having moved it:

\section{2: "It ended up not being the last pass, but the plan was for it to be the final pass. [...] We ended up having to edit in [Adobe] InDesign." (G02)}

Other groups would simply plan for the writing approach to change, effectively transitioning from the collaborative home pattern to one of the other two patterns. Most commonly the repository pattern would be adopted instead, or the group would switch from a parallel or reactive writing strategy (see table 1) to a single writer strategy.

Figure 3 (next page) overall identifies two high-level transition types. Like figure 1, it shows the simplest configuration. Some transitions are closely connected to the progression of the writing process from outline, over text production, to layout, etc., forming a chain of transitions (figure 3, right). Examples include moving from a personal editor to a shared platform to "add collaboration", and then later copying text to a designated layouting application. Other transitions involve moving back and forth between applications, e.g. drafting in a plain text editor. This happens within the aligned artifact ecology as well as between the aligned artifact ecology and members' personal artifact ecologies (figure 3, left).

\section{Categorizing the Transitions}

Functional Transitions

Application transitions motivated by a need for different, better, or more familiar features are common in the empirical data. Such range from smaller features to better general task support. One group discussed how they moved text from Google Docs into MS Word because they found the spell-checker in MS Word to be more accurate than the one in Google Docs, and 

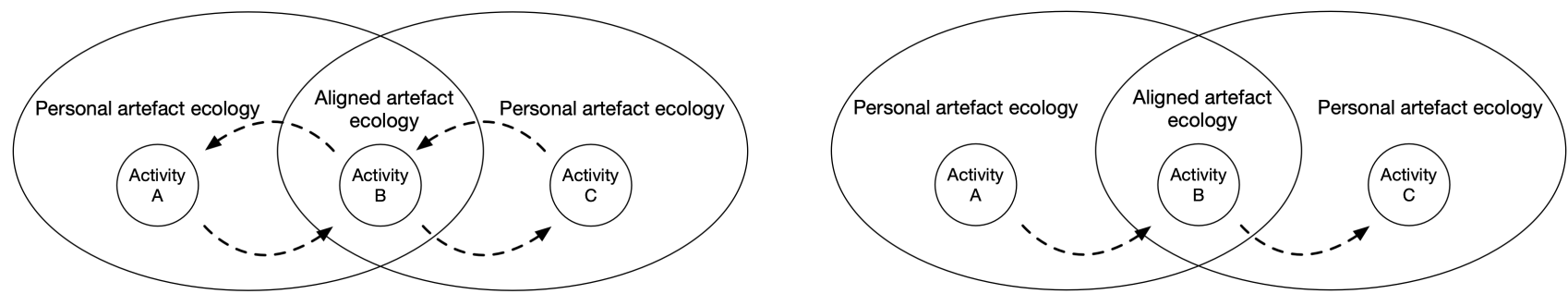

Figure 3: Application transitions within and across ecologies. Left: Back-and-forth transitions. Right: Chaining across applications

others described reference management as a reason for moving from Google Docs into ShareLaTeX. Several projects made a move from Google Docs into ShareLaTeX or Adobe InDesign when needing to do layout and formatting.

\section{Personal Space Transitions}

A majority of the interviewed co-writers describe how they have been starting drafts or moving text outside the master document and/or the collaborative environment in order to produce or revise text on their own. Some discussed seeking solitude to focus on specific parts and/or create a space for working with the text, or for reasons pertaining to selfpresentation, as one respondent who did not want a student of hers to see spelling errors in a draft phase. Another participant explained why he needed a thinking space for drafting text:

3: "I can't really formulate stuff in my head, so I need to write it down and do a few iterations. [...] If I intend to write something that I have not presented to the others yet, and don't know if it makes sense, then it can push me to do it outside, in my own editor." (G11)

Transitions to a personal space may also be motivated by a desire for control, such as not sharing drafts with co-writers before "mak[ing] sure that I've done everything to make it as likely as possible that the feedback I'm getting is useful to $m e$ " (G10). Particularly in cases with independent document control, participants described moving to a separate document to be able to work in the way they preferred or shape the text in a particular way. For participants working in a repository pattern concerns of control or self-presentation influenced the timing of uploads, sometimes resulting in a "huge [Git] commit which [...] other people don't appreciate.” (G12) Issues include the shared text not being up-to-date, and downtime due to using sequential writing to avoid merge conflicts.

\section{Aesthetic and User Experience Transitions}

Transitions to a separate editing environment were also motivated by preferences related to comfort, e.g., being more familiar with MS Word than with Google Docs or needing a "blank slate" (G10) where co-writers' comments would not be visible. A participant stopped using the comment feature in ShareLaTeX because the individual color assigned to each collaborator made it "a real crayon mess" (G10). In some cases such aesthetic experiences of chaos or lack of overview was what drove groups to spend the majority of their writing time in Google Docs despite their plans to do the final formatting in ShareLaTeX, e.g., due to a publication venue requiring the use of a particular template. Many also described Google Docs as more approachable due to its WYSIWYG style. As one participant put it: "ShareLaTeX is for papers and Google Docs is for when working with the others is more important than formatting." (G12) Another participant linked this to disruptions from the additional compilation step in ShareLaTeX:

4: "For instance, making figures, that always breaks things. [...] I sometimes use this online table generator and get the table set up outside, and then paste it into LaTeX when I know that it can compile." (G09)

Similar issues related to experience were distractions and the felt presence of co-writers mediated by the collaborative tool and/or document, in particular in the collaborative home pattern. A frequent example in real-time editing environments is text "jumping" when co-writers add or remove text and the presence of co-writers' cursors within sight and/or blocking the view: "It's very distracting to have a cursor appear and write right above you." (G04). Distractions caused by features of the tool are supplemented by more subtle senses of being surveilled or aspects of the writing process, e.g. being "[a]ffected by seeing others being productive while you're struggling" (G04). Some participants also described how the text "becomes something else" (G05) when moved to a different environment. One example would be that writing in a plain text editor makes the text less set in stone - "it was easier to throw out again, or easier to discard if it was bad" (G01). Whether for reasons pertaining to personal space or user experience, co-writers usually insert text they have produced elsewhere by copy-pasting it into the master document. This is often followed by communicating to the others that something is done and ready for review, frequently via another channel.

\section{Communication Transitions}

Transitions were also motivated by communication needs. Several groups reported that they used multiple ways of reaching co-writers, and would select the application based on the chance of getting a co-writer's attention. One group described starting with Slack, then using the Google Docs chat asking: "Did you see my message?" There were also examples of appropriating the text production environment as an ad hoc messaging channel (real-time synched or not), for instance by locating a co-writers' cursor or text and writing messages at its location, e.g. "Is this okay? Yes, it's fine" (G04) or "STOP CORRECTING I'M NOT FINISHED!" (G02). In many cases this would help facilitate a form of reactive writing. An additional challenge lies in linking communication with in-document content (also discussed by Pearson et al. [55]):

5: "But where it gets difficult is when you need to refer directly to something in the text. [...] Then you make a comment and then you go into Messenger and say: Could you go and check this comment?" (G04) 


\section{Summary - Transitions Within and Across Ecologies}

Transitions between tools are common, with co-writers moving between applications in the aligned ecology as well as applications that only some of them have access to or are familiar with. Applications used for writing are supplemented with additional tools, for producing figures, for doing layout and formatting, for communicating, etc. The same application may play different roles in different collaborations and at different times during the process, mirroring the more individual-focused discussions of Bødker and Klokmose regarding dynamics of artifact ecologies [7]. We have observed transition patterns spanning both personal artifact ecologies and the group's aligned artifact ecology. Whereas motivations for chained transitions are closely coupled to how the shared text and outcome progresses, back-and-forth transitions are linked to a broader set of motivations and needs throughout the process, e.g. self-preservation or avoiding distractions.

Transitions introduce challenges into the writing process. When writers work outside the common documents and applications, their co-writers lose sense of the progress and who is working on what. This is mitigated through ongoing communication and coordination and by maintaining a general sense of responsibility and division of work through the document structure and the text itself (see below). Moving text between documents and applications is usually done by copypasting. The participants experience that differences in how each tool, or each software version, interprets file formats and formatting makes other approaches bothersome and error prone. Finally, transferring text between applications (and documents) introduces two issues frequently discussed by our participants: First, knowing which version is the most up-todate across multiple applications can be difficult (e.g. quote 12), especially when the differences are small as in the case of spell-checking. Second, and related, ensuring that all sections and figures are moved into the right positions, e.g. when finalizing the layout in ShareLaTeX or InDesign.

\section{Text Function(s) in Collaborative Writing}

The above patterns hint at the functions served by the text as a common object during the writing. These echo the three functions of common objects named earlier: The object in its transitional state from materials to product; as epistemic and pointing towards the end product; and as the backbone of communication and coordination (see also [56, 33, 51]). The text is the interim material and outcome of work, a locus of coordination, as well as epistemic and pointing ahead. These functions are further subdivided (see table 1 and below).

Previous work on collaborative writing has emphasized the work around and on the text but less so how the text itself mediates the work as a common object. By explicitly addressing the ways in which the text itself supports the writing - the multiple roles it plays in the writing - we aim to characterize the writing activity as taking place through a common object, and examine how this alternative lens may be used to understand the collaborative writing situation.

The Text as Material and Interim Outcome of Work

The text is naturally in focus for the co-writers as material and product. In addition to molding words and sentence structures, writers continuously interpret additional layers of meaning for information about activities, and where the text is currently at and where it has come from. Through the text they are able to assess how work is progressing, what is there and what has yet to be done, and how finished the text is, etc.

Even if the text exists in multiple versions, of which some older parts may be waiting to be re-used or changed, co-writers (try to) maintain an overview of what the text is. Sometimes the whole text, sometimes sections, exist in multiple places, e.g., when a Google document is moved section-by-section into ShareLaTeX or when a supervisor provides feedback on a printed (old) PDF version while work continues in LaTeX. Different versions or parts in different applications may offer different possibilities for e.g. creating an overview. As an example, it is not as easy to get an idea of the length of a formatted paper in Google Docs as in MS Word or ShareLaTeX, as illustrated here:

6: "[We] needed to know how long the paper was, and we noticed that we were over the limit if we put it in [MS] Word. [...] [The] spacing is somewhat off and different. [...] [We] had to cut almost two pages." (G08)

\section{The Text as a Locus of Coordination and Communication}

Interpretations of the text as an interim outcome of work are facilitated by the its function as an outline (not to be confused with Wang et al.'s [66] outline pattern, which describes how co-writers materially build up the joint text) or a blueprint providing co-writers a material awareness of the form of the text-to-be. Far into the writing process when the originally envisioned structure may have changed, the text continues to serve this function, allowing assessment of progress with respect to the structure of the unfinished document. Likewise, the careful coordination required for keeping track of versions is anchored in the text. Whether in the form of an actual outline in the document, participants' recollections of a meeting, or bullet points in an email, co-writers are enabled to plan how the emerging text will be created and what will happen to it. The common object's function as plan has multiple granularities and abstraction levels, from choice of words to the prospective audience. Sometimes one person is largely in charge of planning and their co-writers' mostly tag along, as in the consulted strategy, while it is a shared and even democratic process in other collaborations. This quote exemplifies how co-writers move back and forth around the text to coordinate:

7: “[Co-writer] took over and made a longer draft, that went through headlines and our vision for the content in detail. Then, as we got a better idea of what the contents would be, that was then adapted." (G04)

This sense of how to go about forming the text in turn enables division of work. This is not a one-off, transient event: The division of work is manifested in the structuring of the work, be it name tags in the document, simply the current placement of co-writers' cursors, or the fact that the main writer has the document on her hard drive for the first week of the writing. We understand from our participants' accounts that they achieve an awareness of the division of work, including what co-writers are currently doing, through the text: 
8: “We've had some [sections] where we've been writing more than the other person. [...] In the end it was pretty clear who was taking care of what." (G05)

The Text as an Epistemic Object

The text's function as an epistemic object is, first, to point ahead. The common object of work is not just the material text in the document. Even before the first character is typed, the (eventual) writing is focused on the text-to-be. This is different from the text as a plan laying out the work to be done. The text's epistemic function is that it embodies the common vision and motivation (see quote 9). The previously described text functions all take their outset in this text-to-be.

The text pointing ahead to its envisioned state allows planning and assessments described above as well as a reflexivity regarding the way the common object is taking shape. The text-to-be is not a fixed vision, as seen in quote 9, but rather one that will change as text is produced. In moving from a common vision to where the manifestation of (and individual ideas about) the object become aligned, co-writers engage in reflection about, with, in, and through the common text:

9: "[At] some point it grew to a hundred pages and then people jokingly called it a book. And then at some point people said: 'Well, why don't we actually make it into a book?'” (G17)

Finally, the text is a common space for reactivity: A place where co-writers respond to each other and can involve each other in their individual processes by reaching out and asking for help. The term "reactivity" is taken from Lowry et al.'s notion of reactive writing, as described in section 2; however, the reactive function of the text is present in any writing strategy, as illustrated in quote 10 where the interviewee acts reactiviely by both looking in the text and poking a co-writer.

10: "[If] you remember: 'Oh, this could be relevant to use in that section!' then just scroll up and check if it is in a bullet point or if the person is currently writing it, and otherwise poke the person and say: 'How about we do this?' " (G04)

This sort of reactivity is possible because the text as an object is held in common, accessible to and accessed by all co-writers at some point or another. By virtue of the text's multiple functions coming together in a shared sense of the common object (quote 10), co-writers are able to maintain a common object in mind as well as on the screen. Of course, the sense of a common vision is sometimes challenged as in quote 11:

11: "There were meetings where everyone was like: 'Wait, so are we saying that [topic] is good or is it bad?' [...] Maybe our initial idea was: 'We'll just write separately and then come together and it will just merge', but I think it didn't and it was all different aspects and we didn't really know what our joint message was." (G16)

In these cases, the role of reflexivity and reactivity in steering and aligning the work become very clear. Writers may in fact apply explicitly agreed upon strategies for reflexivity or reactivity, as when G16 each wrote an abstract to align their thoughts about "what we thought the article should be".

\section{The Common Object of Work}

Not only does the text serve multiple functions in the collaborative work. As we see in quote 12 the degree to which the text is held in common at a certain point in time varies. Motivations such as quote 3 contribute to this.

\section{2: "And then [co-writer] asked me: 'Well, is the current version online?' and I say: 'Yeah sure it's all committed,' but I forgot to push. And then he gives me comments on something and I'm like: Wait, what? No no no, that's not the version I'm in.' " (G15)}

In a parallel, divide and conquer writing situation the common object may only be epistemic at certain moments or, perhaps it is more likely epistemic and coordinative. This means that the common object lies in the future, that the writers share some form of understanding of what the common object will be eventually (see e.g. quote 7). In addition, the writers have some understanding of where their contribution is made, and how this relates to the contribution of the collaborators. Once co-writers consolidate themselves with each other's recent contributions the common object changes and becomes material and manifest. However, the quote below illustrates that this is not always unproblematic and that the choice of tools matters.

13: "One of the big problems with LaTex is that you don't see what has changed. Unless you do a diff, which is hard (...) I actually used software to create a visual PDF, so I could see what the differences between versions were. I've had problems working with people where some of us would suggest like oh, this need to be changed in this way, and then that change was incorporated but not actually in the way that it should have been or the way that the rest of us understood it. But none of us saw it until the paper was at the very final stage." (G08)

During joint writing the text is in common down to paragraphs or even commas and white space. Multiple representations of the text can of course introduce discrepancies, as in Overleaf where the compiled PDF on the screen is not guaranteed to be the same for each co-writer. Whether working together on documents, paragraphs, or commas, these different granularities reveal the multiple levels at which the object of work may be understood and examined, be this by co-writers while collaborating, or by researchers analyzing the collaborative activity. As we have illustrated, the multiple levels are closely tied also to the variety of different tools used, and hence the analysis showcases the effect of working and transitioning within an artifact ecology on the object of work: E.g. checking out a paragraph to use the spell-checker of MS Word means that the particular object cannot be shared meanwhile and needs to be brought back into the common artifact and object.

\section{Summary - Text Function}

In summary, collaborative writing involves text production both together and apart. It involves sharing materials and interim text as objects of work, and importantly it involves collaborating on the epistemic, the joint understanding of the text-to-be. Both the text in production and the many tools around it serve coordination, communication, and division of work. Transitions can occur smoothly, but we have also been 
introduced to hassles such as coupling content to communication, reaching collaborators, and keeping track of versions. This overhead (in CSCW also known as articulation work) can be described with respect to the practical means that writers must utilize (traversing the ecology, implementing manual version control, coordinating through various channels) but can also be described as an effect on the common object and its granularity, in the various functions served by the text.

\section{DISCUSSION}

Our findings highlight ways in which tool use in collaborative writing has changed compared to a couple of decades ago [65]: We corroborate earlier findings [48, 36, 50, 15, 67, 42] on cowriters' habits of and motivations for working in applications intended for individual use, but we have also showcased a wider and more nuanced range of patterns for collaboration which is facilitated (and perhaps spurred on) by collaborators' expansive and overlapping artifact ecologies. While some prior work mentions the inclusion of additional applications in passing [48, 36, 10], only Rossitto et al. [60] have focused on the topic, and unlike us they have not addressed transitions to a personal ecology that is not part of the aligned.

Rossitto et al. [60, p. 156] name Google Drive as an example of a platform that meshes multiple tools to support collaborative writing. They rightly point out that such a pre-defined constellation usually does not stand on its own in co-writers' ecologies, but they leave the example as anecdotal. In many of the groups Google Drive serves as a base in the Collaborative Home pattern until the group makes a chaining transition to an application they find better suited for layouting. This disconnect between different aspects of the text, such as content and layout, means that writers must piece together, and do not always have, a sense of the full interim object of work - for example they may not know how long the paper is, without moving to MS Word (see quote 6) which instead diminishes epistemtic functions of the text because of the asynchronous nature of working in MS Word on separate devices.

This cursory analysis shows how the notion of text function lets us articulate more precisely which aspects of collaborative writing are affected by the different applications in use as well as by co-writers' approach to the writing. It also highlights the significance of examining not just the central hub of the writing, as has typically been done (e.g. [40, 52, 5]), but the full, aligned ecology [60] as well as the interplay with personal ecologies [60]. Such an analysis (in its extended form) is not necessarily intended as a critique of the individual tools as such. Rather, like the presented study, it highlights significant challenges still remaining in supporting collaborative writing that transcends individual applications and devices. As previous work (e.g., $[12,63,52])$ has mainly addressed single-tool scenarios, the problem of supporting transitions as a developed practice in collaborative writing has been left unattended, the consequence being a striking lack of interoperability.

Multiple studies have examined task and application switching from the perspective of recovering from interruptions and supporting more activity-centric configurations [31, 13]. While the current study does not focus on these issues explicitly, it highlights $a$ ) that transitions between applications are a fundamental aspect of collaborative writing (cf. [10]), b) that some of these transitions are prompted by collaborative noise (text jumping, cursor presence, etc.) and a somewhat simple understanding of text production and people's occasional need for personal writing space, and $c$ ) that transitioning between applications is done with copy-pasting and manual re-typing as the primary methods for moving text between applications. In addition to obvious avenues for improving transitions in a current context, such as object embedding for simultaneous editing and forking/merging text, our results lend merit to alternative paradigms, such as document-centric models [37], instrumental interaction [2, 39], or activity-centric perspectives [31,34], that more fully than the application-centric model acknowledge artifact ecologies as the stage for work and other activities, collaborative or individual. Some of our findings parallel issues found in group reading [55] and library group work [44], indicating the relevance of the current study beyond collaborative writing specifically.

How co-writers form their "default" ecologies remains an open area of inquiry, along with how adaptation of the aligned ecology takes place. The work by Rossito et al. [60] is an important take on the contextual nature of this process, but further work is needed to cast light on the influence of mainstream applications and software infrastructures on this. The theoretical contributions of this paper provide a basis for this future work: By focusing on the transitions between artifacts in the ecology, rather than just on the artifacts themselves, we are able to understand shifts between aligned and personal, as well as the shifting borders of the aligned ecology within the potential ecology. Likewise, as demonstrated, our characterization of text functions enables us to examine how transitions and the multiplicity of particular artifact ecologies and co-writers' particular ways of dealing with these impact the common object of work and thereby the continued writing. This framing aims to direct attention to the text as part, rather than merely product $[22,52,70]$, of the collaborative process.

\section{CONCLUSION}

In this work, we have described academic co-writers' motivations for involving multiple tools in their collaborative writing practice. We have used artifact ecologies as a lens for characterizing the transitions that take place within and across aligned and personal ecologies. In doing this, we extend observations from prior research on collaborative writing with more detail while also contributing a categorization of transitions to the literature on artifact ecologies. Based on our findings we also propose the notion of text function to describe how the text as the common object of work is part, not merely product, of the writing; a novel addition to existing taxonomies of collaborative writing.

\section{ACKNOWLEDGEMENTS}

We thank our participants for their essential part in making this work happen. This project has received funding from the European Research Council (ERC) under the European Union's Horizon 2020 research and innovation programme (grant agreement No 740548). 


\section{REFERENCES}

[1] Nancy Allen, Dianne Atkinson, Meg Morgan, Teresa Moore, and Craig Snow. 1987. What Experienced Collaborators Say About Collaborative Writing. Iowa State Journal of Business and Technical Communication 1, 2 (1987), 70-90. DOI :

http://dx.doi.org/10.1177/105065198700100206

[2] Michel Beaudouin-Lafon. 2000. Instrumental Interaction: An Interaction Model for Designing post-WIMP User Interfaces. In Proceedings of the SIGCHI Conference on Human Factors in Computing Systems (CHI 'O0). ACM, New York, NY, USA, 446-453. DOI :

http://dx.doi.org/10.1145/332040.332473

[3] Michael S. Bernstein, Greg Little, Robert C. Miller, Björn Hartmann, Mark S. Ackerman, David R. Karger, David Crowell, and Katrina Panovich. 2015. Soylent: A Word Processor with a Crowd Inside. Commun. ACM 58, 8 (July 2015), 85-94. DOI :

http://dx.doi.org/10.1145/2791285

[4] Jeremy Birnholtz and Steven Ibara. 2012. Tracking Changes in Collaborative Writing: Edits, Visibility and Group Maintenance. In Proceedings of the ACM 2012 Conference on Computer Supported Cooperative Work (CSCW '12). ACM, New York, NY, USA, 809-818. DOI: http://dx.doi.org/10.1145/2145204.2145325

[5] Jeremy Birnholtz, Stephanie Steinhardt, and Antonella Pavese. 2013. Write Here, Write Now!: An Experimental Study of Group Maintenance in Collaborative Writing. In Proceedings of the SIGCHI Conference on Human Factors in Computing Systems (CHI '13). ACM, New York, NY, USA, 961-970. DOI : http://dx.doi.org/10.1145/2470654.2466123

[6] Susanne Bødker. 1990. Through the Interface: A Human Activity Approach to User Interface Design. L. Erlbaum Associates Inc., Hillsdale, NJ, USA.

[7] Susanne Bødker and Clemens Nylandsted Klokmose. 2012. Dynamics in Artifact Ecologies. In Proceedings of the 7th Nordic Conference on Human-Computer Interaction: Making Sense Through Design (NordiCHI '12). ACM, New York, NY, USA, 448-457. DOI : http://dx.doi.org/10.1145/2399016.2399085

[8] Susanne Bødker and Clemens Nylandsted Klokmose. 2016. Dynamics, Multiplicity and Conceptual Blends in HCI. In Proceedings of the 2016 CHI Conference on Human Factors in Computing Systems (CHI '16). ACM, New York, NY, USA, 2538-2548. DOI :

http://dx. doi.org/10.1145/2858036.2858530

[9] Susanne Bødker, Henrik Korsgaard, and Joanna Saad-Sulonen. 2016. 'A Farmer, a Place and at Least 20 Members': The Development of Artifact Ecologies in Volunteer-based Communities. In Proceedings of the 19th ACM Conference on Computer-Supported Cooperative Work \& Social Computing (CSCW '16). ACM, New York, NY, USA, 1142-1156. DOI: http://dx.doi.org/10.1145/2818048.2820029
[10] Tom Boellstorff, Bonnie Nardi, Celia Pearce, and T. L. Taylor. 2013. Words with Friends: Writing Collaboratively Online. interactions 20, 5 (Sept. 2013), 58-61. DOI : http://dx.doi.org/10.1145/2501987

[11] Richard E. Boyatzis. 1998. Transforming Qualitative Information: Thematic Analysis and Code Development. SAGE Publications, Inc.

[12] Cornelia Brodahl and Nils Kristian Hansen. 2014. Education Students' Use of Collaborative Writing Tools in Collectively Reflective Essay Papers. Journal of Information Technology Education: Research 13, 1 (2014), 91-120. DOI : http://dx.doi .org/10.28945/1960

[13] Gaston R. Cangiano and James D. Hollan. 2009. Capturing and Restoring the Context of Everyday Work: A Case Study at a Law Office. In Human Centered Design, Masaaki Kurosu (Ed.). Springer Berlin Heidelberg, Berlin, Heidelberg, 945-954.

[14] Martha Ann Carey and Mickey W. Smith. 1994. Capturing the Group Effect in Focus Groups: A Special Concern in Analysis. Qualitative Health Research 4, 1 (1994), 123-127. DOI : http://dx.doi .org/10.1177/104973239400400108

[15] Teresa Cerratto and Henrry Rodriguez. 2002. Studies of Computer Supported Collaborative Writing. Implications for System Design. In Cooperative Systems Design. IOS Press, Amsterdam, 139-154.

[16] Andrew L. Cohen, Debra Cash, Michael J. Muller, and Curtis Culberson. 1999. Writing Apart and Designing Together. In CHI '99 Extended Abstracts on Human Factors in Computing Systems (CHI EA '99). ACM, New York, NY, USA, 198-199. DOI: http://dx.doi.org/10.1145/632716.632840

[17] Andrew M. Dearden and Janet Finlay. 2006. Pattern Languages in HCI: A Critical Review. Human-Computer Interaction 21 (2006), 49-102.

[18] David Dearman and Jeffery S. Pierce. 2008. It's on My Other Computer!: Computing with Multiple Devices. In Proceedings of the SIGCHI Conference on Human Factors in Computing Systems (CHI '08). ACM, New York, NY, USA, 767-776. DOI : http://dx.doi.org/10.1145/1357054.1357177

[19] C. A. Ellis and S. J. Gibbs. 1989. Concurrency Control in Groupware Systems. SIGMOD Rec. 18, 2 (June 1989), 399-407. DOI: http://dx.doi.org/10.1145/66926.66963

[20] Douglas C. Engelbart. 1984. Collaboration Support Provisions in AUGMENT. In Proceedings of the 1984 AFIPS Office Automation Conference (OAC'84 Digest). $51-58$.

[21] Yrjö Engeström. 2014. Learning by Expanding: An Activity-Theoretical Approach to Developmental Research (2 ed.). Cambridge University Press. DOI : http://dx.doi .org/10.1017/СВ09781139814744 
[22] Gijsbert Erkens, Gellof Kanselaar, Maaike Prangsma, and Jos G. M. Jaspers. 2002. Using tools and resources in computer supported collaborative writing. In CSCL. 389-398.

[23] Sarah Evans, Katie Davis, Abigail Evans, Julie Ann Campbell, David P. Randall, Kodlee Yin, and Cecilia Aragon. 2017. More Than Peer Production: Fanfiction Communities As Sites of Distributed Mentoring. In Proceedings of the 2017 ACM Conference on Computer Supported Cooperative Work and Social Computing (CSCW '17). ACM, New York, NY, USA, 259-272. DOI : http://dx.doi.org/10.1145/2998181.2998342

[24] Robert S. Fish, Robert E. Kraut, and Mary D. P. Leland. 1988. Quilt: A Collaborative Tool for Cooperative Writing. In Proceedings of the ACM SIGOIS and IEEECS TC-OA 1988 Conference on Office Information Systems (COCS '88). ACM, New York, NY, USA, 30-37. DOI : http://dx.doi.org/10.1145/45410.45414

[25] James H. Frey and Andrea Fontana. 1991. The group interview in social research. The Social Science Journal 28, 2 (1991), 175 - 187. DOI :

http://dx.doi.org/10.1016/0362-3319(91)90003-M

[26] Sebastian Gehrmann, Lauren Urke, Ofra Amir, and Barbara J. Grosz. 2015. Deploying AI Methods to Support Collaborative Writing: A Preliminary Investigation. In Proceedings of the 33rd Annual ACM Conference Extended Abstracts on Human Factors in Computing Systems (CHI EA '15). ACM, New York, NY, USA, 917-922. DOI :

http://dx.doi.org/10.1145/2702613.2732705

[27] GitHub, Inc. 2019. Built for developers. (2019). Retrieved March 24, 2019 from https://github.com/

[28] Google. 2019. Create persuasive/impactful/meaningful documents. (2019). Retrieved March 24, 2019 from https://www. google. com/docs/about/

[29] Jörg M. Haake and Brian Wilson. 1992. Supporting Collaborative Writing of Hyperdocuments in SEPIA. In Proceedings of the 1992 ACM Conference on Computer-Supported Cooperative Work (CSCW'92). Association for Computing Machinery, New York, NY, USA, 138-146. DOI :

http://dx.doi.org/10.1145/143457.143472

[30] Christian Heath and Paul Luff. 1996. Documents and Professional Practice: "Bad" Organisational Reasons for "Good" Clinical Records. In Proceedings of the 1996 ACM Conference on Computer Supported Cooperative Work ( $C S C W$ '96). Association for Computing Machinery, New York, NY, USA, 354-363. DOI : http://dx.doi.org/10.1145/240080.240342

[31] D. Austin Henderson, Jr. and Stuart Card. 1986. Rooms: The Use of Multiple Virtual Workspaces to Reduce Space Contention in a Window-based Graphical User Interface. ACM Trans. Graph. 5, 3 (July 1986), 211-243. DOI : http://dx.doi.org/10.1145/24054.24056
[32] Thomas Herrmann, Marcel Hoffmann, Isa Jahnke, Andrea Kienle, Gabriele Kunau, Kai-Uwe Loser, and Natalja Menold. 2003. Concepts for Usable Patterns of Groupware Applications. In Proceedings of the 2003 International ACM SIGGROUP Conference on Supporting Group Work (GROUP '03). Association for Computing Machinery, New York, NY, USA, 349-358. DOI : http://dx.doi.org/10.1145/958160.958216

[33] Morten Hertzum. 1999. Six Roles of Documents in Professionals' Work. Springer Netherlands, Dordrecht, 41-60. DOI :

http://dx.doi.org/10.1007/978-94-011-4441-4_3

[34] Steven Houben, Paolo Tell, and Jakob E. Bardram. 2014. ActivitySpace: Managing Device Ecologies in an Activity-Centric Configuration Space. In Proceedings of the Ninth ACM International Conference on Interactive Tabletops and Surfaces (ITS '14). ACM, New York, NY, USA, 119-128. DOI:

http://dx.doi.org/10.1145/2669485.2669493

[35] Heekyoung Jung, Erik Stolterman, Will Ryan, Tonya Thompson, and Marty Siegel. 2008. Toward a Framework for Ecologies of Artifacts: How Are Digital Artifacts Interconnected Within a Personal Life?. In Proceedings of the 5th Nordic Conference on Human-computer Interaction: Building Bridges (NordiCHI '08). ACM, New York, NY, USA, 201-210. DOI : http://dx.doi .org/10.1145/1463160.1463182

[36] Hee-Cheol (Ezra) Kim and Kerstin Severinson Eklundh. 2001. Reviewing Practices in Collaborative Writing. Computer Supported Cooperative Work (CSCW) 10, 2 (01 Jun 2001), 247-259. DOI : http://dx. doi .org/10.1023/A: 1011229212323

[37] Laurent Kirsch, Jean Botev, and Steffen Rothkugel. 2012. Snippets and Component-Based Authoring Tools for Reusing and Connecting Documents. Journal of Digital Information Management 10, 6 (2012), 399-409. http://www.dline.info/fpaper/jdim/v10i6/6.pdf

[38] Jenny Kitzinger. 1995. Qualitative Research: Introducing focus groups. BMJ 311, 7000 (1995), 299-302. DOI : http://dx.doi.org/10.1136/bmj . 311.7000.299

[39] Clemens Nylandsted Klokmose and Michel Beaudouin-Lafon. 2009. VIGO: Instrumental Interaction in Multi-surface Environments. In Proceedings of the SIGCHI Conference on Human Factors in Computing Systems (CHI '09). ACM, New York, NY, USA, 869-878. DOI : http://dx.doi.org/10.1145/1518701.1518833

[40] Jenell Krishnan, Andrew Cusimano, Dakuo Wang, and Soobin Yim. 2018. Writing Together: Online Synchronous Collaboration in Middle School. Journal of Adolescent \& Adult Literacy 62, 2 (2018), 163-173. DOI : http://dx.doi.org/10.1002/jaal. 871

[41] Steinar Kvale. 2007. Doing interviews. Sage. DOI: http://dx.doi.org/10.4135/9781849208963 
[42] Ida Larsen-Ledet and Henrik Korsgaard. 2019. Territorial Functioning in Collaborative Writing. Computer Supported Cooperative Work (CSCW) (08 May 2019). DOI :

http://dx.doi.org/10.1007/s10606-019-09359-8

[43] Paul Benjamin Lowry, Aaron Curtis, and Michelle René Lowry. 2004. Building a Taxonomy and Nomenclature of Collaborative Writing to Improve Interdisciplinary Research and Practice. The Journal of Business Communication (1973) 41, 1 (2004), 66-99. DOI : http://dx.doi.org/10.1177/0021943603259363

[44] Dana McKay and George Buchanan. 2014. On the Other Side from You: How Library Design Facilitates and Hinders Group Work. In Proceedings of the 26th Australian Computer-Human Interaction Conference on Designing Futures: The Future of Design (OzCHI '14). ACM, New York, NY, USA, 97-106. DOI : http://dx. doi .org/10.1145/2686612.2686625

[45] Alex Mitchell, Ilona Posner, and Ronald Baecker. 1995. Learning to Write Together Using Groupware. In Proceedings of the SIGCHI Conference on Human Factors in Computing Systems (CHI '95). ACM Press/Addison-Wesley Publishing Co., New York, NY, USA, 288-295. DOI : http://dx.doi.org/10.1145/223904.223941

[46] Bonnie A Nardi and Vicki O'Day. 1999. Information ecologies: Using technology with heart. Mit Press.

[47] Christine M. Neuwirth, Ravinder Chandhok, David S. Kaufer, Paul Erion, James Morris, and Dale Miller. 1992. Flexible Diff-Ing in a Collaborative Writing System. In Proceedings of the 1992 ACM Conference on Computer-Supported Cooperative Work (CSCW '92). Association for Computing Machinery, New York, NY, USA, 147-154. DOI:

http://dx.doi.org/10.1145/143457.143473

[48] Christine M. Neuwirth, David Kaufer, Ravinder Chandhok, and James H. Morris. 2000. Computer Support for Distributed Collaborative Writing: A Coordination Science Perspective. In Coordination Theory and Collaboration Technology. Lawrence Erlbaum Assoc.

[49] Davide Nicolini, Jeanne Mengis, and Jacky Swan. 2012. Understanding the Role of Objects in Cross-Disciplinary Collaboration. Organization Science 23, 3 (2012), 612-629. DOI :

http://dx.doi.org/10.1287/orsc. 1110.0664

[50] Sylvie Noël and Jean-Marc Robert. 2004. Empirical Study on Collaborative Writing: What Do Co-authors Do, Use, and Like? Computer Supported Cooperative Work (CSCW) 13, 1 (01 Mar 2004), 63-89. DOI : http://dx.doi.org/10.1023/B:COSU.0000014876.96003.be

[51] Kenton O'Hara, Mark Perry, Abigail Sellen, and Barry Brown. 2002. Exploring the Relationship between Mobile Phone and Document Activity during Business Travel. Springer London, London, 180-194. DOI : http://dx.doi.org/10.1007/978-1-4471-0665-4_12
[52] Judith S. Olson, Dakuo Wang, Gary M. Olson, and Jingwen Zhang. 2017. How People Write Together Now: Beginning the Investigation with Advanced Undergraduates in a Project Course. ACM Trans. Comput.-Hum. Interact. 24, 1, Article 4 (March 2017), 40 pages. DOI : http://dx.doi.org/10.1145/3038919

[53] Antti Oulasvirta and Lauri Sumari. 2007. Mobile Kits and Laptop Trays: Managing Multiple Devices in Mobile Information Work. In Proceedings of the SIGCHI Conference on Human Factors in Computing Systems (CHI '07). ACM, New York, NY, USA, 1127-1136. DOI : http://dx.doi .org/10.1145/1240624.1240795

[54] Overleaf. 2019. LaTeX, Evolved. (2019). Retrieved March 24, 2019 from https://www. overleaf.com/

[55] Jennifer Pearson, Tom Owen, Harold Thimbleby, and George R. Buchanan. 2012. Co-reading: Investigating Collaborative Group Reading. In Proceedings of the 12th ACM/IEEE-CS Joint Conference on Digital Libraries (JCDL '12). ACM, New York, NY, USA, 325-334. DOI :

http://dx.doi .org/10.1145/2232817.2232876

[56] Mark Perry and Duncan Sanderson. 1998. Coordinating joint design work: the role of communication and artefacts. Design Studies 19, 3 (1998), 273 - 288. DOI : http://dx. doi .org/10.1016/S0142-694X(98)00008-8

[57] I. R. Posner and R. M. Baecker. 1992. How people write together. In Proceedings of the Twenty-Fifth Hawaii International Conference on System Sciences, Vol. 4. 127-138. DOI :

http://dx. doi.org/10.1109/HICSS. 1992.183420

[58] Dimitrios Raptis, Jesper Kjeldskov, Mikael B. Skov, and Jeni Paay. 2014. What is a digital ecology? Theoretical foundations and a unified definition. Australian Journal of Intelligent Information Processing Systems 13, 4 (2014), 5.

[59] Mike Robinson. 1993. Design for unanticipated use..... In Proceedings of the Third European Conference on Computer-Supported Cooperative Work 13-17 September 1993, Milan, Italy ECSCW '93, Giorgio de Michelis, Carla Simone, and Kjeld Schmidt (Eds.). Springer Netherlands, Dordrecht, 187-202. DOI: http://dx.doi.org/10.1007/978-94-011-2094-4_13

[60] Chiara Rossitto, Cristian Bogdan, and Kerstin Severinson-Eklundh. 2014. Understanding Constellations of Technologies in Use in a Collaborative Nomadic Setting. Computer Supported Cooperative Work (CSCW) 23, 2 (01 Apr 2014), 137-161. DOI : http://dx.doi .org/10.1007/s10606-013-9196-4

[61] Steve Sawyer, Kevin Crowston, and Rolf T. Wigand. 2014. Digital assemblages: evidence and theorising from the computerisation of the US residential real estate industry. New Technology, Work and Employment 29, 1 (2014), 40-56. DOI: http://dx.doi . org/10.1111/ntwe. 12020 
[62] Pål Sørgaard. 1989. A Framework for Computer Supported Cooperative Work. DAIMI Report Series 17, 253 (March 1989), 20. DOI :

http://dx.doi.org/10.7146/dpb.v17i253.7609

[63] Jaime Teevan, Shamsi T. Iqbal, and Curtis von Veh. 2016. Supporting Collaborative Writing with Microtasks. In Proceedings of the 2016 CHI Conference on Human Factors in Computing Systems (CHI'16). Association for Computing Machinery, New York, NY, USA, 2657-2668. DOI :

http://dx.doi.org/10.1145/2858036.2858108

[64] Josh Tenenberg, Wolff-Michael Roth, and David Socha. 2016. From I-Awareness to We-Awareness in CSCW. Comput. Supported Coop. Work 25, 4-5 (Oct. 2016), 235-278. DOI :

http://dx.doi.org/10.1007/s10606-014-9215-0

[65] Dakuo Wang. 2016. Exploring and Supporting Today's Collaborative Writing. Ph.D. Dissertation. University of California, Irvine.

[66] Dakuo Wang, Judith S. Olson, Jingwen Zhang, Trung Nguyen, and Gary M. Olson. 2015. How Students Collaboratively Write using Google Docs. In iConference 2015 Proceedings. http://hdl.handle.net/2142/73736

[67] Dakuo Wang, Haodan Tan, and Tun Lu. 2017. Why Users Do Not Want to Write Together When They Are
Writing Together: Users' Rationales for Today's Collaborative Writing Practices. Proc. ACM Hum.-Comput. Interact. 1, CSCW, Article 107 (Dec. 2017), 18 pages. DOI :

http://dx.doi.org/10.1145/3134742

[68] Weigang Wang and Jörg M. Haake. 1998. Flexible Coordination with Cooperative Hypertext. In Proceedings of the Ninth ACM Conference on Hypertext and Hypermedia: Links, Objects, Time and Space-Structure in Hypermedia Systems (HYPERTEXT '98). Association for Computing Machinery, New York, NY, USA, 245-255. DOI :

http://dx.doi.org/10.1145/276627.276654

[69] Thomas Wynn. 1993. Layers of thinking in tool behavior. Tools, Language and Evolution (1993), 389-406.

[70] Soobin Yim, Dakuo Wang, Judith Olson, Viet Vu, and Mark Warschauer. 2017. Synchronous Collaborative Writing in the Classroom: Undergraduates' Collaboration Practices and Their Impact on Writing Style, Quality, and Quantity. In Proceedings of the 2017 ACM Conference on Computer Supported Cooperative Work and Social Computing (CSCW'17). ACM, New York, NY, USA, 468-479. DOI :

http://dx.doi.org/10.1145/2998181.2998356 